\title{
Familia e inserción social
}

\section{M ayda Álvarez Suárez}

Centro de Investigaciones Psicológicas y Sociológicas (CIPS).

Calle B, \#352, esquina a 15. 10400 Vedado. La H abana. Cuba

Fecha de recpción: noviembre de 1995

\section{Resumen}

Profundos y trascendentales cambios han tenido lugar en la estructura y funcionamiento de la familia en todo el mundo y Cuba no ha estado ajena a los mismos.

El presente artículo analiza las variaciones que la inserción a diferentes sectores y grupos sociales introduce en el funcionamiento familiar.

Se inicia la caracterización de las familias cubanas con datos acerca del tamaño y composición de los hogares. Se destaca la disminución del tamaño medio de los núcleos, el aumento de los hogares encabezados por mujeres y la disminución de las diferencias del tamaño de las familias entre zonas urbanas y rurales a partir de 1959.

La función biosocial de la familia es abordada a través del análisis de los procesos de formación y disolución de las familias y de sus aportes a la reproducción de la población.

Un estudio realizado en una muestra representativa nacional de familias obreras y de trabajadores intelecturales, urbanas y completas, sirve de base para el análisis de las variaciones que la inserción social de los miembros adultos introduce en el cumplimiento de las funciones económica y cultural de la familia. El artículo concluye con reflexiones acerca del papel de la familia en la sociedad cubana actual.

Palabras clave: inserción social y familia, funciones familiares, tamaño y composición de las familias, matrimonio, divorcio, fecundiad, trabajo doméstico, comunicación familiar.

Abstract. Family and social insertion

D eep changes have taken place in the family structure and function everywhere in the world and in Cuba. This article deals with the changes induced by insertion in different sectors and social groups. It examines variations in family composition and dimension as well as the biosocial function of this institution through statistical and survey data.

Key words: family functions, social insertion, family composition, domestic labour.

\section{Sumario}

Tamaño y composición de las familias

Procesos de formación y disolución de las familias

La ampliación de la familia y su aporte a la reproducción de la población
Inserción social y funciones familiares

Función económica de la familia

Función cultural de la familia

Reflexiones finales

Bibliografía 
$\mathrm{H}$ ablar y escribir sobre el tema de la familia nunca resulta fácil, pues aunque todos estamos de acuerdo en que ella es, al mismo tiempo, institución y un grupo social responsable de importantes funciones y cargado de gran afectividad; las distintas personas - según su edad, sexo y grupo social - pueden concebirla y vivenciarla de muy diversos modos.

En la actualidad es muy frecuente escuchar que la familia está en crisis. EI incremento de los índices de divorcio, de maternidad temprana y soltera, de hogares uniparentales, de uniones consensuales, hacen pensar en la desintegración de esta institución.

En Cuba han tenido también lugar debates en torno a esta cuestión, y estudiosos sobre el tema, basándonos en los resultados de investigaciones realizadas, nos hemos pronunciado al respecto en un reciente trabajo (M . Álvarez y otros, 1994).

$M$ ás adelante, retomaremos al gunos de esos criterios, pero antes resulta imprescindible saber de cuál familia estamos hablando: ¿cuántas familias viven en Cuba?, ¿cómo están compuestas?, ¿qué las diferencia?, ¿cómo cumplen sus funciones?

\section{Tamaño y composición de las familias}

No contamos con datos muy actualizados acerca de la cantidad y la composición de las familias cubanas, pues nuestras dificultades económicas no han permitido la realización de un nuevo censo de población. Son los datos del Censo de Población y Vivienda de 1981 y los de la Encuesta de Presupuesto Familiar de 1987 los que entregan una respuesta aproximada de cuántas familias viven en nuestro país y de qué tipo de familia predomina en cuanto a su composición.

En 1981 existían en nuestro territorio 2 millones 351 mil núcleos particulares ${ }^{1}$ en los que residía el $99,6 \%$ de la población; el $70 \%$ de ellos ubicados en zonas urbanas.

La familia cubana tiende a ser pequeña. El cambio más significativo entre 1953 y 1981 fue la importante disminución del tamaño promedio de los núcle os de 4,9 a 4,1 personas como promedio nacional, y el hecho de que las grandes diferencias existentes en 1953 entre las zonas urbanas y rurales en cuanto al tamaño de los núcleos se atenuaron. N o obstante siguen existiendo una menor proporción de núcleos de 1 a 2 personas y una mayor presencia de núcleos de 7 y más personas en las zonas rurales.

Esta importante disminución del tamaño promedio de los núcleos se asocia a los planes de construcción de viviendas impulsados por el Gobierno revolucionario, sobre todo en zonas rurales, y a los bajos niveles de fecundidad que

1. Los llamados «núcleos particulares» se diferencian del concepto de familia en que incluyen los hogares de una sola persona y aquellos en los que residen personas que no tienen lazos de parentesco. 
han tenido lugar en el país básicamente a partir del segundo lustro de los años setenta.

Una fuente más reciente sobre el promedio de consumidores por núcleo arroja que esta cifra es de 3,5 a nivel nacional. Aunque este dato no siempre coincide con el número de residentes reales de la vivienda, resulta coherente con la tendencia a la que nos hemos venido refiriendo.

En cuanto a su composición predominan las familias formadas por la pareja y sus hijos, que residen en una unidad de vivienda, y otras formas de familias nucleares (matrimonios solos; el padre o la madre y sus hijos).

Las familias nucleares fueron en 1981 el 53,7\% del total de núcleos particulares. Sin embargo, las familias extendidas, que representan sólo el 32,5\% de los núcleos, son importantes por el volumen de población que en ellas reside: $41,9 \%$ de la población total.

Se ha venido observando también un aumento de los hogares encabezados por mujeres entre 1953 y 1981, donde la proporción de mujeres jefas dentro del total de jefes de núcleo se ha duplicado de un $14 \%$ a un $28 \%$.

D icho en otros términos, en 1953 por cada 100 mujeres de 15 años y más, 9,6 de ellas eran jefes de núcleo. En 1981 esta relación ascendió a 19,7 por cada 100. Es posible, no obstante, que la proporción de hogares dirigidos por mujeres resulte mayor si se tiene en cuenta la tendencia cultural de asociar la jefatura de los hogares con el género masculino. En 1981 las áreas urbanas registraban un 34,1\% de jefas de hogares, mientras que en las rurales este porcentaje era sólo del 14\% (M . Álvarez y S. Catasús, 1994).

D e las jefas mujeres se encontraban sin cónyuges el $64 \%$ de los casos, ya sea por viudez (25,6\%) o por divorcio o separación (19,3\%).

Un estudio nacional realizado por el grupo de familia del CIPS (Centro de Investigaciones Psicológicas y Sociológicas) sobre 3.685 familias urbanas, integradas por la pareja adulta (casada o unida) y al menos un hijo soltero entre 12 y 19 años, constató que los tamaños predominantes son 4 y 5 personas, en este orden de importancia; y que el número modal es dos hijos solteros. En general, si el hijo adolescente o joven tiene hermanos en edades que varían entre 12 y 19 años; pero en una proporción importante el hermano es mucho menor (infante o escolar), debiendo los padres atender las necesidades y la formación de los hijos en distintas fases del desarrollo.

Las familias nucleares constituían el $59,1 \%$ de las familias estudiadas. En 40 de cada 100 familias, los hijos adolescentes y jóvenes conviven no sólo con sus padres y hermanos solteros, sino con una amplia variedad de parientes, entre los que destacan en primer lugar sus abuelos (19,5\%) y en menor medida hermanos o hermanas casados $(9,7 \%)$ y otras personas no emparentadas ( $7 \%$ ) (I. Reca, 1989).

La diversidad de tipos de familias extendidas constatadas en dicho estudio indica que no existe un patrón de preferencia común en la convivencia familiar. M ás bien refleja la insuficiente disponibilidad de vivienda, pues no muestra una situación de elección o preferencia sino de compartir la vivienda en la medida que existen familiares dispuestos a acogerlos. 
Para muchos adolescentes y jóvenes su familia tiene tres generaciones. Ello hace mucho más compleja la dinámica del grupo y diversifica las influencias educativas.

Paralelamente a los cambios referidos en la estructura de la familia, se han producido también transformaciones en su funcionamiento.

Resulta necesario tener en cuenta que no todas las familias cumplen de forma homogénea sus funciones, variados factores introducen diferenciaciones en las propias familias y en la forma en que ellas dan cumplimiento a dichas funciones: su estructura; sus condiciones objetivas de vida; la inserción de los miembros adultos a distintas clases, capas y grupos de la sociedad; la incorporación o no de la mujer al trabajo asalariado; las diferencias culturales y territoriales del lugar donde se habita; la etapa del ciclo vital en la que se encuentre.

Así, un importante estudio realizado con una muestra representativa de 1.125 familias completas, urbanas, obreras y de trabajadores intelectuales con hijos adolescentes y jóvenes mostró que las familias de trabajadores intelectuales poseen mejores condiciones de vida (vivienda, equipamiento, ingreso per cápita, nivel escolar) que las familias obreras, y éstos se convierten a su vez en factores favorecedores para el ejercicio de las funciones familiares.

D e igual forma, la inserción de la mujer al trabajo asalariado contribuye a una mejor distribución de las tareas domésticas en el hogar y a enriquecer la comunicación con sus hijos (I. Reca y otros, 1989).

D etengámonos entonces en los cambios ocurridos en el ejercicio de las funciones familiares en la familia cubana.

\section{Procesos de formación y disolución de las familias}

Los procesos de formación y disolución de las parejas son de vital transcendencia para la función biosocial de la familia. Esta función que incluye las relaciones sociales y afectivas de la pareja y su conducta reproductiva, está estrechamente vinculada a los cambios demográficos y tiene importantes efectos sociales en cuanto a lo que a reproducción de la población se refiere.

En Cuba el matrimonio sigue siendo la forma más extendida de constituir familia. Si bien entre 1970 y 1988 se registró un paulatino descenso del número de matrimonios y un ligero incremento de las uniones consensuales, especialmente en la población joven, las tasas brutas de nupcialidad en los años transcurridos de la década de los noventa se incrementan.

En 1981 la tasa bruta de nupcialidad fue de 7,5 por mil habitantes; 9,5 en 1990; 17,7 en 1992; 12,4 en 1993 y 10,7 en 1994.

No obstante, no es posible desconocer el importante cambio en la forma de constituir familia en los jóvenes registrado entre 1970 y 1981 . Pongamos un ejemplo, si en 1970, por cada 100 varones casados entre 15 y 19 años, fueron censados 76 en unión consensual; en 1981 esta relación fue de 189 unidos por cada 100 casados.

La importancia que viene ganando la unión consensual se evidencia también dentro del grupo de mujeres de edad fértil. En 1970 se reportaron 58,4 
mujeres unidas por cada 100 casadas, relación que ascendió a 60 y 81,7 en 1981 y 1987 respectivamente. Es decir, en Cuba en 1987 por cada cinco mujeres casadas entre 15 y 49 años, existían al menos cuatro unidas consensualmente (T. Pedroso, 1993).

Un reciente estudio de la consensualidad en los jóvenes muestra la existencia de diferentes tipos de uniones: la unión asociada a la crisis económica, la unión moderna, la unión tradicional y la unión asociada a dificultades en la relación de pareja (S. G onzález y T. Alfonso, 1995).

Sin embargo, a pesar de la importancia que viene ganando la unión consensual para muchas de las personas, el matrimonio legal constituye una aspiración.

O tra característica a destacar en la formación de parejas es el rejuvenecimiento de la nupcialidad cubana: el promedio de edad al primer matrimonio disminuyó a 18,4 años, el más bajo de América Latina.

Aunque no se cuenta con datos nacionales, varios estudios indican que la convivencia en familias extendidas y el limitado acceso a la vivienda son dos características típicas y complementarias de las condiciones de vida de la pareja y de familias jóvenes, especialmente en las grandes ciudades (I. Reca y M . Alvarez, 1989).

También se han estudiado las características psicosociales en parejas jóvenes que van a contraer matrimonio, constatándose falta de preparación en sus miembros para la vida familiar. Esta falta de preparación se evidencia en indicadores como la existencia de dificultades para la comunicación interpersonal; el hecho de casarse alentados por una imagen poco realista de lo que es el matrimonio y la convivencia; la falta de constructividad en la solución de los conflictos y el no aprovechamiento de la etapa del noviazgo para profundizar en el conocimiento mutuo (M. Álvarez y M. Díaz, 1989).

La ruptura de las uniones - legales o consensuales- ha aumentado también en los últimos años. Las estadísticas de divorcio reflejan que si en 1961 por cada mil personas sólo 0,6 rompieron su matrimonio, en 1989 esta relación resultó de 3,6 por mil; en 1991, de 4,1; en 1993, de 6,0, y en 1994, de 5,2 por mil. En otras palabras, en 1994 por cada 100 matrimonios realizados tuvieron lugar 48,5 divorcios.

También es importante tomar en consideración la población que se declara separada. Esta categoría aumentó durante la década de los ochenta. Si tomamos como ejemplo la población femenina entre 15 y 49 años, se observa que la proporción de mujeres en edad fértil separada varió del 5,1\% al 10,3\% entre 1981 y 1987.

El proceso de ruptura de las uniones adquiere una mayor transcendencia en los jóvenes menores de 20 años; el $50 \%$ de los casados con estas edades disuelven el vínculo durante los dos primeros años de matrimonio.

Son diversos los factores que se relacionan con el aumento de las rupturas: el arribo de las cohortes de nacidos en los sesenta a las edades de mayor frecuencia de formalización de matrimonios; el incremento de la participación de la mujer en la vida social y económica del país, las dificultades en las condi- 
ciones de vida con las cuales los jóvenes inician su convivencia, además de la poca preparación que aún éstos tienen para el matrimonio y la vida en familia.

\section{La ampliación de la familia y su aporte a la reproducción de la población}

$\mathrm{N}$ uestra población crece a un ritmo lento, lo que depende fundamentalmente de los bajos niveles de natalidad que se registran desde 1978.

Entre 1961 y 1964 la fecundidad en Cuba experimentó un transitorio aumento, alcanzando niveles de 2,30 hijos por mujer; en el último lustro de los setenta comenzó un sistemático descenso (aunque con oscilaciones ocasionales) que desde 1978 ubica a la fecundidad cubana por debajo del nivel de reemplazo de la población. En 1994 ña TG F fie de 1.5 hijos por mujer, mientras la TBR fue de 0,7 hijos por mujer.

C aracterizan estas variaciones de los niveles de la fecundidad en Cuba la homogeneidad territorial y su acentuado rejuvenecimiento. La primera se evidencia en que las diferentes provincias del país experimentaron cambios similares y es precisamente en la zona rural donde se han producido las disminuciones más acentuadas.

Por otra parte, el rejuvenecimiento de la fecundidad se manifiesta por el aumento significativo del aporte del grupo de 15 a 19 años, cuyos niveles de fecundidad en todos estos años se han mantenido altos. Es decir, «la reducción de los niveles de la fecundidad en Cuba se ha producido básicamente a expensas de las mujeres del extremo superior del período fértil» (M . Álvarez y S. C atasús, 1994).

Si bien en la actualidad la baja fecundidad no es considerada negativa en tanto no presiona sobre la economía, a mediano y largo plazo los efectos sobre el envejecimiento de la población serán desfavorables.

\section{Inserción social y funciones familiares}

¿Q ué variaciones introducen al funcionamiento de la familia la pertenencia de sus miembros adultos a diferentes capas y grupos sociales?

El importante estudio nacional ya citado con familias completas, urbanas, con hijos adolescentes y jóvenes (I. Reca y otros, 1989) nos permitió comparar a los dos grupos cuantitativamente más importantes de familias en nuestra sociedad: familias obreras y de trabajadores intelectuales.

El análisis se centrará fundamentalmente en dos importantes funciones: la económica y la cultural.

\section{Función económica de la familia}

La función económica tiene lugar a través de la convivencia en el hogar común y la administración de la economía doméstica. Comprende las actividades de abastecimiento y consumo, las cuales permiten la satisfacción de las necesidades individuales y familiares. 
También las actividades de mantenimiento de la familia, que incluyen todos los aportes del trabajo que realizan sus miembros en el hogar y que denominamos tareas o quehaceres domésticos, así como el cuidado de los niños, de los enfermos y las vías y contactos con las instituciones de salud, educación, etc. (I. Reca y otros, 1991).

El aporte de la familia cubana a la reposición de la fuerza de trabajo mediante la realización del conjunto de tareas domésticas dirigidas a garantizar el cre cimiento y el mantenimiento de sus miembros es muy significativo. La difícil situación económica en que vive el país en la actualidad, donde escasean los recursos materiales y la existencia y el desarrollo de servicios de apoyo al hogar son limitados, hace que la vida cotidiana de las familias sea mucho más compleja, ocupando dicha función económica gran parte del tiempo y de los esfuerzos de los miembros de la familia, principal mente de la mujer.

El estudio nacional realizado en 1988 y 1989 con 1.200 familias (600 familias obreras y 600 familias de trabajadores intelectuales) aportaba ya importantes datos al respecto.

Se constató un predominio en la familia cubana $(59,4 \%)$ de un modelo de distribución de tareas domésticas marcadamente desigual, «modelo tradicional», en el que la mujer deviene responsable máxima de las obligaciones domésticas (M aría del C. Caño, 1989). Este hecho, a pesar de poseer un alto grado de difusión en la sociedad cubana, no se presenta de manera homogénea en toda la población estudiada, resulta dependiente de:

a) La inserción socioclasista de la familia, en la medida en que las de la clase obrera reflejan una desiguald dad mayor en el plano de las relaciones familiares que las familias de trabajadores intelectuales. En estas últimas existe un mayor número ubicadas en los modelos transicional y perspectivo de distribución de tareas domésticas ( $21,6 \%$ y 5,9\% respectivamente) por un 14,0 y $1,9 \%$ en las familias obreras.

b) La actividad laboral y el tipo de ocupación de la mujer. La proporción de familias de mujeres amas de casas en las que impera el modelo tradicional de distribución de tareas domésticas es mayor que la de las familias de muje res trabajadoras asalariadas. En el caso de las últimas, las de mujeres dirigentes constituyen el reflejo de un evidente tránsito hacia formas superiores de distribución y organización del trabajo doméstico. En este grupo de familias se constató la mayor proporción $(10,2 \%)$ de las que poseen un «modelo perspectivo», mientras que en las familias de mujeres ocupadas como obreras y trabajadoras de los servicios se apreciaron las proporciones menores $(3,4 \%$ y $1,2 \%$ respectivamente).

Las características de la ocupación de la mujer imprimen un mayor grado de diferenciación a la distribución del trabajo doméstico que el que resulta de la inserción socioclasista de la familia, en la medida en que se aprecian diferencias significativas con mayor magnitud al interior del grupo de familias en las que la mujer pertenece a la capa de los trabajadores inte- 
lectuales. Como resultado de estas diferencias, las familias en las que la mujer es empleada administrativa manifiestan un comportamiento más parecido al de las familias de la clase obrera.

D e modo general, del conjunto de variables mencionadas que condicionan el tipo de relaciones intrafamiliares que se establecen en la realización del trabajo doméstico, el vínculo laboral de la mujer resultó la variable de mayor incidencia.

c) El al to nivel de escolaridad de la mujer contribuye adecuadamente al logro de una distribución más equitativa del trabajo doméstico: la mayor proporción de familias en las que impera el modelo perspectivo de distribución se observa en aquellas en que la mujer tiene un nivel de escolaridad muy alto. Estas mujeres son, a su vez, las que en mayor proporción logran emplear eficazmente su tiempo libre.

La alta proporción de mujeres que declara un alto nivel de satisfacción con la desigual distribución del trabajo doméstico y el criterio de «avanzada», en cuanto a su distribución, referido con mayor frecuencia en las familias estudiadas, evidencian la existencia de una contradicción entre el plano de las ideas de los individuos y el de realidad cotidiana de la familia cubana. Dicha contradicción debe contribuir perspectivamente al funcionamiento de la familia estimulando el perfeccionamiento de las relaciones intrafamiliares en el proceso de la distribución de roles en el terreno del trabajo doméstico.

La distribución más equitativa del trabajo doméstico, es decir, «el modelo perspectivo», resulta característico básicamente para aquellas familias en las que la pareja realiza un número menor de tareas domésticas (hasta 5 tareas).

La desigual distribución del trabajo doméstico a nivel de pareja, influye negativamente en la reproducción de un patrón negativo en la participación del hijo en tareas domésticas que tiende a ser baja, en sentido general, aunque se diferencia significativamente por sexo y por edad, en tanto las hembras reportan un nivel significativamente mayor de participación en dichas tareas.

El bajo nivel de distribución de tareas domésticas que predomina en las relaciones entre padres e hijos, se manifiesta con independencia del vínculo laboral y del nivel de escolaridad de la mujer, de las diferencias sociales y del tipo de familia: nuclear o extendida.

La invariabilidad del fenómeno referido apunta hacia una pauta generalizada en los padres de no adjudicar a los hijos responsabilidades y tareas en el hogar, ni hacerlos participar consciente y activamente en la dinámica familiar cotidiana. La inexistencia de una relación de dependencia entre la participación del hijo en tareas domésticas y el nivel de solicitud de los padres (padre y madre) de realizar alguna tarea en la casa confirman la lógica de la aseveración inicial.

Si a esto añadimos que los padres perciben que realizan solicitudes de participación a los hijos con una frecuencia mayor que la que refieren éstos 
últimos, queda finalmente establecido el bajo nivel de exigencia que matiza las relaciones de los padres con los hijos en esta área del funcionamiento familiar.

La evaluación que realizan la madre y el hijo sobre la participación de éste en las tareas domésticas resultó marcadamente diferente, debido a que el hijo tiende a sobreestimar la magnitud de la contribución que realiza en la familia en la ejecución de las tareas del hogar.

\section{Función cultural de la familia}

La función cultural incluye el conjunto de actividades educativas y culturales, de superación, las recreativas y de tiempo libre de la familia, las cuales contribuyen a la formación de intereses, valores y al desarrollo de la personalidad de cada uno de los miembros de la familia. En ella tienen un importante papel las relaciones de comunicación e interacción entre padres, madres e hijos y todo el conjunto de relaciones intrafamiliares (I. Reca y otros, 1991). D e la misma forma que en el ejercicio de la función económica, es la mujer quien desempeña el papel más importante en el desarrollo de las actividades y el tipo de relaciones correspondientes a esta función.

Este hecho se evidencia en los resultados relativos al proceso de comunicación en la familia. Tanto en los temas como en las características y en las funciones de la comunicación se comprobó que las madres conversan más fre cuentemente con sus hijos que los padres y comparativamente son ellas quienes ejercen mayor control y regulación sobre la conducta de los hijos y las que les expresan con mayor frecuencia afecto y vivencias experimentadas en las relaciones interpersonales.

Según la percepción de los hijos, las madres utilizan métodos más adecuados en su educación, ya que cuando ellos analizan los patrones de sanciones utilizadas por los padres para regular su conducta, valoran a sus madres como más persuasivas que a sus padres.

Tanto en los aspectos de la comunicación como a patrones de sanciones se refiere, se constató la existencia de mayor coherencia ente las respuestas del hijo a la madre que en las del hijo al padre. Ello revela diferencias en la percepción de las relaciones, lo que constituye un indicador indirecto de la existencia de mayores dificultades en la comunicación entre padre e hijo.

Si bien resulta positivo que la madre esté desempeñando un papel importante en las actividades y en las relaciones que inciden de manera directa en la formación de los hijos, el hecho de ser prácticamente sólo ella la máxima responsable de las mismas tiene un aspecto negativo, y es que trae aparejado un rol complementario de padre poco activo, que se relaciona menos con sus hijos y delega gran parte de su responsabilidad en su compañera.

En las familias estudiadas sí existe preocupación por los hijos y atención sobre todo, en cuanto a los asuntos de la escuela y sus estudios actuales y futuros, lo que se constató en que estos aspectos constituyen los asuntos más frecuentes de conversación entre los padres y sus hijos (sobre todo entre las madres 
y sus hijos). Además, entre las actividades de tiempo libre de los adultos, se constata la ayuda al hijo en las tareas.

El hecho de que la escuela y los estudios futuros constituyen el tema primordial en la conversación de los padres con sus hijos resulta esperable y además importante. La mayoría de los adolescentes y jóvenes investigados son estudiantes y tienen ante sí - a corto o más largo plazo- la selección de la futura profesión, lo que exige de ellos reflexión y un nivel de resultados académicos que hagan factibles sus aspiraciones; mucho más teniendo en cuenta que la elevación actual del nivel de exigencia en la educación trae aparejado un mayor reclamo a la familia de participación en el control y en la estimulación de la actividad de estudio de sus hijos. La gran mayoría de los adultos declara emplear métodos persuasivos al sancionar a sus hijos. En ello coinciden ambos miembros de las parejas.

Los patrones de sanciones no persuasivos (agresividad física, verbal, etc.) se constataron en el 33\% de la muestra, y los emplea uno de los miembros de la pareja, mientras que el otro siempre es persuasivo.

Es posible que en esas parejas suceda que ambos padres se complementan en cuanto a si uno es más punitivo el otro es más persuasivo. Pero quizás se trate de que uno es quien esté a cargo de la educación del hijo cotidianamente, mientras el otro viene de manera esporádica a aplicar las sanciones cuando ya se agotan las fuerzas del primero.

Esto se relaciona mucho con sancionar al hijo amenazándolo con decírselo a su papá, que es su patrón de sanción típico cuando el hijo es varón y menor de 15 años. Sin embargo, estos hijos no consideran esta situación como una sanción sino como paso intermedio para ser luego castigado por el padre, dada la imposibilidad de la madre de hacerlo.

Este proceder no es efectivo, pues posterga en el tiempo la sanción y diluye la responsabilidad de sancionar al hijo en el otro miembro de la pareja.

En sentido general, se constataron serias dificultades en la educación de los varones, por cuanto:

a) A ellos se les sanciona más que a las hembras con métodos no persuasivos (agresiones físicas y verbales).

b) Con ellos se conversa menos: siendo las madres las personas que más conversan con sus hijos, ellas tratan más «habitualmente» con las hembras que con los varones la mayoría de los temas, mientras que con éstos últimos se conversa «siempre que es necesario». Aunque los padres en general conversan poco, cuando lo hacen con los varones es para tratar asuntos de la escuela, de las relaciones de noviazgo o de cuestiones materiales; sin embargo, con las hijas hembras conversan significativamente más sobre las relaciones interpersonales con compañeros y amigos y sobre política internacional.

c) No se les prepara para enfrentar con independencia la satisfacción de determinadas necesidades cotidianas (alimentación, lavado de ropas, etc.); lo que se evidencia en la bajísima participación en las tareas domésticas. 
La información obtenida según la edad de los hijos en las variables relativas a la comunicación y a los patrones de sanciones no muestran una regularidad en su comportamiento que permita llegar a conclusiones según los grupos de edades estudiados. Estos datos muestran que el tratamiento de los padres a los hijos no es diferenciado según la edad deéstos, sino que más bien depende de las características de los padres en las diferentes familias.

D iversos indicadores muestran una situación más favorable con respecto a las relaciones con los hijos y a la atención que se brinda a determinados aspectos de su educación en las familias de ISC intelectual con respecto a las familias de ISC obrera. Así se constató que:

a) El nivel más alto de apertura de la comunicación es característico de las familias de FTI, el cual está también fuertemente asociado a la alta escolaridad de los padres.

b) Padres y madres de FTI conversan significativamente con sus hijos con más frecuencia que los padres de FO sobre un conjunto de temas, entre los cuales se encuentran algunos señalados como deficitarios para la muestra en general, estos temas son: sexualidad, política, relaciones interpersonales con compañeros de estudio o trabajo, literatura, asuntos de la escuela y estudios futuros.

c) Con respecto a las frecuencias de la comunicación, no se constataron diferencias en cuanto a la función regulativa en una u otra familia según su inserción socioclasista, sin embargo, al gunos de sus indicadores como son brindar orientación y ayuda a los hijos, señal arle los errores que cometen y planificar las salidas y las vacaciones conjuntamente, tienen un comportamiento más favorable en cuanto a su frecuencia en las FTI.

d) En cuanto a la función afectiva, se constató una diferencia a favor de las FTI $(30,91 \%)$ con respecto a las FO $(25,85 \%)$, por cuanto madres e hijos de estas familias señalan hablan significativamente con más frecuencia sobre lo que les agrada o desagrada, sobre los asuntos importantes para el hijo y sobre los problemas personales de las madres. Por otra parte, los hijos de estas familias perciben a sus madres como más cariñosas que los hijos de FO .

e) Si bien la mayoría de los adultos declara emplear métodos persuasivos a la hora de sancionar a sus hijos ( $80 \%$ ), es en las familias de trabajadores intelectuales donde se presentan las mayores coincidencias entre la percepción de los hijos y sus padres al respecto. Al parecer, las familias obre ras tienden a ofrecer una imagen más positiva que la realidad de cómo sancionar a sus hijos, ya que en estas familias ambos padres coinciden en afirmar que utilizan métodos persuasivos más que en las familias de trabajadores intelectuales.

\section{Reflexiones finales}

Los datos aportados por las investigaciones sobre la familia en Cuba - a al gunos de los cuales ya hemos hecho referencia- muestran que esta institución en 
nuestras condiciones sigue teniendo un papel esencial en cuanto a su aporte a la reposición de la fuerza de trabajo y en el cuidado de los niños y ancianos.

Por otra parte, no obstante la expansión de la educación estatal y social, la responsabilidad de la familia cubana en la educación y socialización de niños, adolescentes y jóvenes no ha decrecido. Ello sigue siendo fundamental en aspectos tan importantes como la formación de normas de convivencia y patrones de conducta social. Tampoco ha podido ser relevada de sus funciones psicológicas como espacio de comunicación personal, de transmisión de afecto y seguridad, además de preservación y transmisión de nuestras tradiciones y valores culturales (M . Álvarez y otros, 1994).

Para la población cubana la familia sigue siendo una de las áreas más importantes de su vida: cual quier investigación que se realice encaminada a indagar acerca de los valores, motivaciones e intereses de las personas constata que ellas expresan en un primer plano la necesidad y el deseo de vivir en familia y la perciben como fuente de cariño y afecto. En la conducta también se refleja esta importancia: las personas continúan casándose o uniéndose establemente, es decir, constituyendo y viviendo en familia.

L as redes de parentesco, sobre todo aquellas establecidas con la familia de origen, tienen un papel muy importante en nuestra realidad cotidiana, en aspectos fundamentales como el apoyo económico, en la realización de tareas domésticas y en el cuidado de niños y ancianos. Todo lo anteriormente expresado, nos permite afirmar que la familia en Cuba no ha perdido importancia ni mucho menos se está desintegrando; sí está atravesando por un proceso de cambio, de tránsito hacia un modelo de relaciones más democrático, en el cual la solidaridad, la responsabilidad compartida, la ayuda y el respeto mutuos y la afectividad constituyen las bases fundamentales sobre las cuales dicho modelo va edificándose en medio de múltiples contradicciones.

\section{Bibliografía}

Álvarez, M ayda; D íAz, M . Características de la comunicación en parejas que van a contraer matrimonio. CIPS-ACC, 1989. Informe de investigación.

Álvarez, M ayda; C ATASús, S. «La familia cubana: composición, estructura y funciones». Boletín. ICAP, La H abana, 1994.

ÁlvAREZ, M ayda y otros. La familia cubana: cambios, actualidad y retos. D epartamento de Estudios sobre Familia. CIPS-ACC, La H abana, 1994.

C AÑ o, M aría del C. «Función Económica dela Familia». En: Caracterización del modo de vida de las familias obreras y de trabajadores intel ectuales y cumplimiento de su función formadora. CIPS-ACC, 1989. Informe de investigación.

Comité Estatal de Estadísticas. O ficina $\mathrm{N}$ acional del Censo. Censo de Población y Viviendas, 1981. República de Cuba, La H abana, 1985. Vol. 16.

Com ITÉ Estatal De Estadísticas. D irección de D emografía: «C aracterísticas de los núcleos y la familia». En: Encuesta D emográfica N acional. La H abana, 1987.

Comité EstatAL DE EstAdísticas. Encuesta Nacional de Fecundidad, 1987. Instituto de Investigaciones Estadísticas, La H abana, 1991. 
Departam ento de Estudios so bre Familia. Impactos del Período Especial en la familia cubana. CIPS-ACC, La H abana, 1992. Informe de investigación.

González SuseT; Alfon so, T. La consensualidad en los jóvenes. CIPS-CIT M A, La H abana, 1995. Informe de investigación.

In STITUTO N ACIONAL DE IN VESTIGACIONES ESTAD ÍSTICAS. D atos aportados acerca del promedio de consumidores por núcleos hasta diciembre de 1993. La H abana, 1994.

O ficina N acion al de Estadísticas. Anuario D emográfico de Cuba, 1994. M inisterio de Economía y Planificación. La H abana, Cuba, 1995.

Ped Roso, Teresa. Familia: Transición demográfica y situación de la mujer en Cuba. Instituto de Investigaciones Estadísticas. Comité Estatal de Estadísticas. La H abana, 1993.

RECA, Inés. Algunas características demográficas y sociológi cas de las familias urbanas, completas con hijos adolescentes y jóvenes. CIPS-AC C, La H abana, 1989.

ReCA, Inés; Álvarez, M. y colaboradores. La familia cubana hoy. CIPS-ACC, Editado por SIT-M IN FAR, La H abana, 1989.

- Análisis de las investigaciones sobre la familia cubana. 1970-1987. Editorial Ciencias Sociales, La H abana, 1990.

RECA, Inés y otros. Caracterización del modo de vida de las familias obreras y de trabajadores intelectuales y cumplimiento desu función formadora. CIPS-ACC, La H abana, 1989. Informe de investigación.

- La familia en el ejercicio de susfunciones. D epartamento de Estudios sobre Familia. CIPS-ACC, La H abana, 1991. 